\title{
Correction of the effects of acoustic heterogeneity on thermoacoustic tomography using transmission ultrasound tomography
}

Xing Jin, Lihong V. Wang

Xing Jin, Lihong V. Wang, "Correction of the effects of acoustic heterogeneity on thermoacoustic tomography using transmission ultrasound tomography," Proc. SPIE 6086, Photons Plus Ultrasound: Imaging and Sensing 2006: The Seventh Conference on Biomedical Thermoacoustics, Optoacoustics, and Acousto-optics, 60860W (6 March 2006); doi: 10.1117/12.645104 


\title{
Correction of the effects of acoustic heterogeneity on thermoacoustic tomography using transmission ultrasound tomography
}

\author{
Xing Jin, Lihong V. Wang* \\ Optical Imaging Laboratory, Department of Biomedical Engineering \\ Texas A\&M University, 3120 TAMU, College Station, Texas 77843-3120
}

\begin{abstract}
The effects of acoustic heterogeneities on thermoacoustic tomography (TAT) are examined and corrected. One assumption made in the existing reconstruction algorithms for thermoacoustic tomography is that biological tissue is acoustically homogeneous. In medical imaging applications, this assumption can cause blurring and distortion in the reconstructed images. This degradation of image quality can be compensated for by using an approximate distribution of the acoustic speed in the tissue. A method based on transmission ultrasound tomography, which is compatible with our thermoacoustic imaging setup, is developed to correct those effects. Experiments verify the validity of this method. The technique can be used to improve the image quality of thermoacoustic tomography.
\end{abstract}

Keywords: Thermoacoustic tomography, transmission ultrasound tomography, acoustic heterogeneity, acoustic speed, time-of-flight.

\section{INTRODUCTION}

Microwave-induced TAT has been shown to be capable of overcoming some of the limitations of conventional ultrasound imaging [1] [2] [3]. In TAT imaging, pulsed microwave energy is delivered to the biological tissue through a waveguide. The absorbed energy in the tissue induces a short period of temperature change and, consequently, generates thermoacoustic waves through thermoelastic expansion. We use an ultrasonic transducer to record the thermoacoustic waves. The obtained thermoacoustic waves are then used to reconstruct TAT images. Because tumorous tissue and normal biological tissue have a significant difference in water content, and most other biological tissues have microwave absorption coefficients somewhere between these two, we can obtain high contrast in TAT imaging. TAT also has high resolution due to the short wavelength of the generated thermoacoustic waves. Because of its high sensitivity to water content in the tissue and its high resolution, TAT has the potential to be used to detect tumors at an early stage.

Existing reconstruction algorithms for TAT assume that the acoustic speed in biological tissues is constant. If the acoustic speed variation in biological tissue is small, we can obtain TAT images with good image quality by adjusting the average acoustic speed. In medical imaging applications, however, this assumption can not be always satisfied. To obtain better image quality, we need to consider the effects of acoustic heterogeneities in the tissue. For example, in the case of breast imaging, parenchyma, connective tissue and fatty tissue all have different acoustic speeds [4], and the difference in these speeds can be as great as $10 \%$. If we assume constant acoustic speed, the thermoacoustic signal will be back-projected without position correction, and this will cause distortion and blurring in the reconstructed image. This degradation of image quality is more serious for small tumor detection. Ideally, we could use an exact acoustic

\footnotetext{
* To whom all correspondence should be addressed. Telephone: 979-847-9040; Fax: 979-845-4450; Email: LWang@tamu.edu; URL: http://oilab.tamu.edu.
} 
speed distribution to compensate for these effects. In reality, no such technology is available. A previous study has shown that we can use an approximate acoustic speed distribution to improve the image quality of TAT [5], but we must first find an appropriate approximate measurement of the acoustic speed distribution in biological tissues. We have known that ultrasound transmission tomography can provide good quality speed-of-sound distributions in tissues [6] [7] In addition, ultrasound transmission tomography is compatible with our current experimental setup. Therefore, we use ultrasound transmission tomography to obtain the acoustic speed distributions. The purpose of this paper is to present our preliminary results on correcting the effects of acoustic heterogeneities on thermoacoustic tomography.

\section{METHODS}

The experimental setup for TAT has been shown in [1] and [3]. Figure 1 shows only a schematic graph of the scanning system for the combined TAT and ultrasound transmission tomography. The phantom sample was made by inserting a piece of porcine muscle in porcine fat. The whole sample was immersed in mineral oil and placed on a base in the X-Y plane. The acoustic speed in the porcine muscle is around $1.50 \sim 1.58 \mathrm{~mm} / \mu \mathrm{s}$; the acoustic speed in the porcine fat is around $1.42 \sim 1.45 \mathrm{~mm} / \mu \mathrm{s}$ [4]. Two unfocused transducers (V323, Panametrics Inc.) were used in the experiments. The water content in the porcine muscle is similar to that in tumorous tissues. The central frequency of the transducers was $2.25 \mathrm{MHz}$, and the diameter of the active element was $6 \mathrm{~mm}$. Both of the transducers were mounted on a mechanical arm.

In the ultrasound transmission tomography, both of the transducers were used. One was used as a transmitter to send ultrasonic pulses; the other one, on the opposite side of the transmitter, was used to receive the ultrasonic pulses. In the experiments, the mechanical arm, propelled by a stepping motor, first scanned in a fan-beam to cover the tissue sample. Then, the center of the fan-beam, propelled by a second stepping motor, scanned the target region circularly. A pulse-receiver (PR 5072, Panametrics Inc.) was used to send and receive the ultrasonic pulses. The thermoacoustic signals were recorded by a data acquisition card (CS14200, Gage Inc.). For image reconstruction, we first measured the time-of-flight perturbation by cross-correlating between the ultrasound wave transverse through the medium with, and without, the to-be-imaged object. The time-of-flight perturbation measurements were then used to reconstruct the speedof-sound image of the tissue by a filtered back-projection method [8].

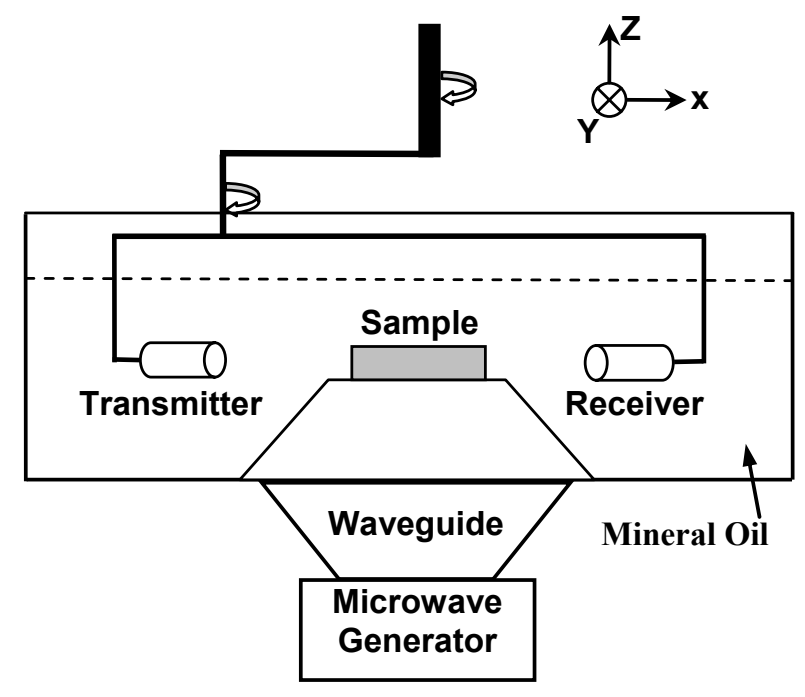

Figure 1: Schematic graph of the scanning system of the combined imaging system. 


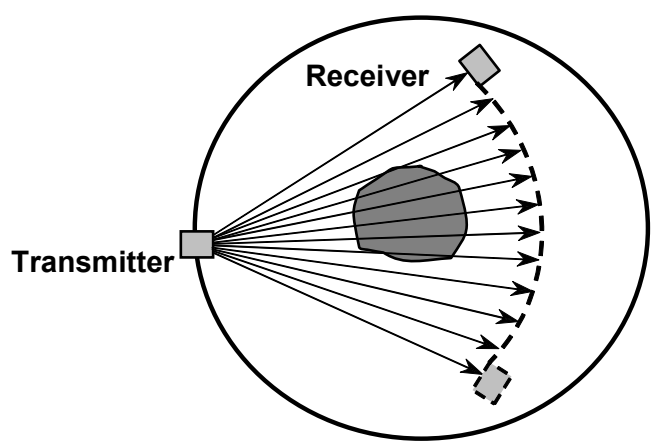

Figure 2: Scanning geometry for ultrasound transmission tomography in top view.

In the thermoacoustic tomography, pulsed microwave radiation was used to irradiate the sample. We did not need a transmitter; therefore, only one of the transducers was used for data collection. In the experiment, the ultrasonic transducer, controlled by a stepping motor, scanned the tissue sample circularly. The frequency of the microwave was 3 $\mathrm{GHz}$. At this frequency, the penetration depth in fat was $9 \mathrm{~cm}$, and the penetration depth in muscle was $1.2 \mathrm{~cm}$. The peak power of the microwave pulse was estimated to be $2 \mathrm{~kW}$, and the estimated total energy of the microwave pulse was about $1 \mathrm{~mJ}$. The microwave radiation level was below the safety requirement. The reconstruction of the thermoacoustic tomography was similar to the commonly used one [3], except that in the forward problem, the integration surface is not an ideal one because it becomes a perturbed integration surface. Every point source on this surface had the same time of flight between the source and receiver. In the back-projection, the time taken for the acoustic wave to propagate from the source to the detector was not obtained by the geometrical distance divided by the acoustic speed; instead, it became integrated along the path connecting the source and the detector.

For the speed compensation, we first made an initial estimate of the image by constructing a TAT image by adjusting the average acoustic speed and then incorporating the acoustic speed distribution obtained by ultrasound transmission tomography into the TAT data. We then constructed a TAT image that was more accurate than the initial estimate.

\section{RESULTS}

The performance of the proposed method was further investigated using a phantom experiment. In the experiment, we assumed weak refraction, and, therefore, only first-order speed distortion was considered for speed compensation. Usually we obtain better TAT images by adjusting the average acoustic speed in the biological tissue. This method works well when we try to detect large absorbers. From our experiment, however, we found that the image quality of TAT is limited when we try to detect small absorbers in a heterogeneities background. Therefore, our experiment focuses on improving the ability of TAT to detect small tumors in a heterogeneous background.

In this phantom experiment, we used porcine fat and muscle because the difference between their acoustic speeds is relatively large. The acoustic speeds of porcine muscle and fat are in the range of acoustic speeds for breast tissue [4]. Figure 3(a) shows the phantom sample used in the experiments. Figure 3(b) gives the speed-of-sound image obtained. We obtained the speed-of-sound image by using ultrasound transmission tomography. We can see that ultrasound transmission tomography does not work well to image small speed heterogeneities. 


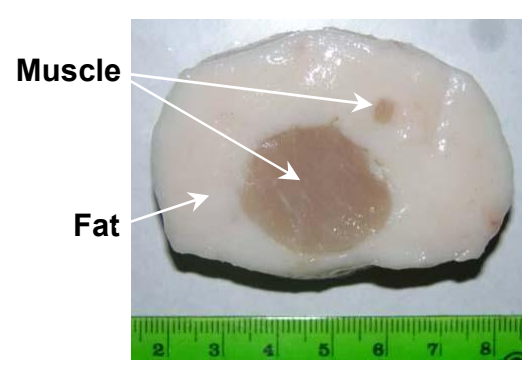

(a)

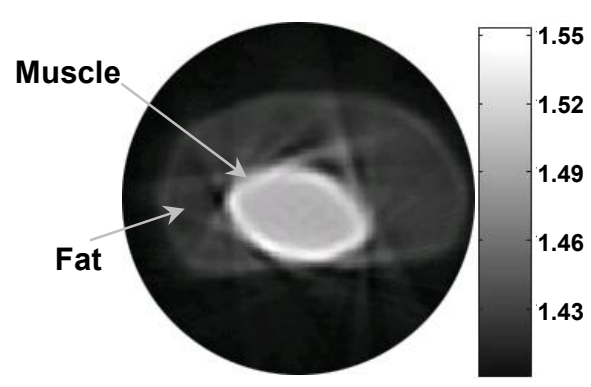

(b)

Figure 3: (a) Photograph of the phantom used in the experiment; (b) speed-of-sound image.

We compared the results of our method with the results of the most commonly used method in Fig. 4. First, we reconstructed the focused TAT image by adjusting the average acoustic speed of the tissue to obtain the sharpest boundary for the porcine fat and muscle, as shown in Fig. 4(a). We got a blurred image for the small absorber as marked by white arrows. The boundary of the small absorber was also not clear. The TAT image obtained by the proposed method with speed compensation is shown in Fig. 4(b). The boundary of the small absorber was denoted by white arrows. The boundary of the smaller absorber obtained by the proposed method was sharper than that obtained by the method that ignored acoustic heterogeneities. The blurring of the small absorber was also not as serious in Fig. 4(b) as in Fig. 4(a).

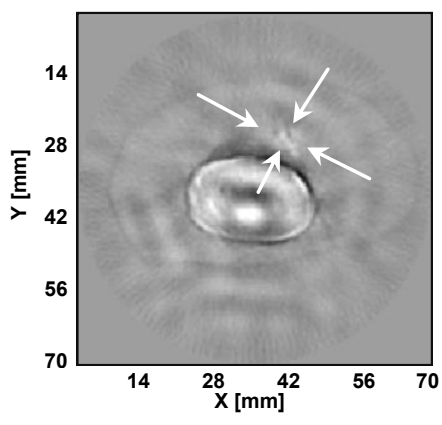

(a)

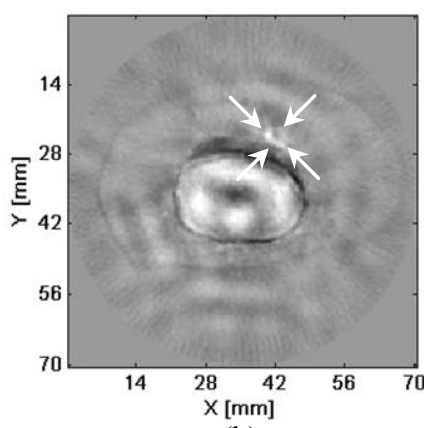

(b)

Figure 4: (a) TAT image with acoustic speed compensation from adjusting average speed; (b) TAT image with speed compensation from using acoustic speed distribution obtained by ultrasound transmission tomography.

The TAT image based on the proposed method has been shown to have better image quality than that obtained by the method that ignores acoustic speed heterogeneities.

\section{CONCLUSIONS}

We presented a method to correct the effects of acoustic heterogeneities on TAT images using an approximate acoustic speed distribution. In the study, we focused on weakly scattering biological tissue. In our experiment, we have demonstrated that ultrasound transmission tomography can provide good quantitative images of the acoustic speed distribution of a phantom sample and that those acoustic speed images have sufficient quantitative accuracy for worthwhile compensation of the effects of acoustic heterogeneities on TAT. The results obtained by our method show 
that acoustic speed compensation by ultrasound transmission tomography is a feasible approach for improving the resolution and contrast of TAT, thus potentially increasing the capability of TAT to detect small tumors in acoustically heterogeneous backgrounds.

\section{REFERENCE}

1. G. $\mathrm{Ku}$ and L.-H. Wang, "Scanning microwave-induced thermoacoustic tomography: signal, resolution, and contrast," Med, Phys., vol. 28, 4-10 (2001).

2. Y. Xu and L.-H. Wang, "Reconstructions in limited-view thermoacoustic tomography", Med. Phys., vol. 31, no. 3, Mar., (2003)

3. M. Xu and L.-H. Wang, "Time-domain reconstruction for thermoacoustic tomography in a spherical geometry," IEEE Trans, on Med. Imag. 21 (7), 814-822 (July 2002).

4. Francis A. Duck, "Physical properties of tissue: a comprehensive reference book", Academic press (1990)

5. Y. Xu and L. V. Wang, "Effects of acoustic heterogeneity in breast thermoacoustic tomography", IEEE Trans. Ultrason., Ferro., and Freq. Co.l, vol. 50, no. 9, 1134-1146 (2003)

6. G. H. Clover and J. C. Sharp, " Reconstruction of ultrasound propagation speed distribution in soft tissue: time-offlight tomography”, IEEE Trans. on Sonics and Ultrasonics, vol. SU-24, no. 4, 229-234 (1977)

7. J. F. Greenleaf, and R. C. Bahn, "Clinical imaging with transmissive ultrasonic computerized tomography", IEEE Trans. on Biomed. Eng., vol. 28, no. 2, 177-185 (1981)

8. A. C. Kak, M. Slaney, Principles of computerized tomographic imaging, IEEE Press, (1988) 